\title{
MRI visibility of the anterolateral ligament and the deep structures of the iliotibial tract
}

\author{
Michael Liebensteiner ${ }^{1}$, Armin Runer ${ }^{2 *}$, Christof Kranewitter ${ }^{3}$, Philipp Nachtigal ${ }^{4}$, Johannes Giesinger ${ }^{5}$, \\ Dietmar Dammerer ${ }^{1}$ and Benjamin Henninger $^{3}$
}

\begin{abstract}
Purpose: The visualization of potentially injured anatomical structures is crucial. Lately the anterolateral ligament (ALL) and the deep structures of the iliotibial tract (ITT) have been of increased clinical interest because of their role as important lateral stabilizers of the knee. The aim of this study was to assess the visibility of the ALL and the deep structures of the ITT using MRI. Good intra- and inter-observer reproducibility was hypothesized.
\end{abstract}

Methods: Knee MRI data from patients without ligamentous lesions were retrospectively analyzed by two radiologists at two time points using axial and coronal sequences. The visibility of the different parts of the ALL (femoral, meniscal and tibial part) and of the deep ITT, namely the deep attachments of the ITT to the distal femur and capsulo-osseous layer of the ITT, were determined on a binary (yes/no) basis.

Results: Seventy-one cases (42 men, 29 women) were studied. Inter-observer agreement was high. Cohen's kappa was 0.97 for the tibial part of the ALL and 0.76 for the femoral part. For the deep attachments of the ITT to the distal femur Cohen's kappa was 0.94. For each of the investigated parameters absolute agreement between the observers was at least $88 \%$. Regarding intra-observer agreement Cohen's kappa was 0.62 for the femoral part of the ALL and 0.85 for the tibial part of the ALL. For the deep attachments of the ITT to the distal femur Cohen's kappa was 0.94 . For each investigated parameter absolute agreement between the two time points was at least $83 \%$.

Conclusions: The presence of the anterolateral structures of the knee can be determined with substantial interand intra-observer agreement using MRI examination. This is applicable for both the ALL and the deep ITT.

Level of evidence: Diagnostic study - Level III.

Keywords: Anterolateral ligament, lliotibial tract, lliotibial band, Anterior cruciate ligament, Knee stability, Magnetic resonance imaging

\section{Background}

Since 2013 there has been an increased interest in the anterolateral extra-articular soft-tissue structures of the knee and their importance in controlling rotatory knee stability. In particular, the anterolateral ligament (ALL) was popularized as an important ligamentous stabilizer

\footnotetext{
* Correspondence: armin.runer@tirol-kliniken.at

2Deptartment of Trauma Surgery, Medical University of Innsbruck, Innsbruck, Austria

Full list of author information is available at the end of the article
}

of the anterolateral knee [4]. This triggered several investigations of ALL traceability during cadaver dissection $[4,5,11,29,31,32,36]$ and the biomechanical function of the ALL [19, 26, 29, 33, 35]. Several studies have dealt with MRI visibility of the ALL $[9,12-14,20,21,24,30$, 37]. Nonetheless, little is known about validity and reproducibility in assessing the ALL $[7,13,14,37]$. Ferretti et al. [7] reported inter- and intra-observer reliabilities of the ALL in different MRI imaging parameters ranging between $0.69-1$ and $06-1$, respectively. Helito et al. [13] 
studied the validity of MRI in detecting the ALL using an anatomic evaluation as reference and reported an intra-observer reliability between 0.77 and 0.93 .

While the above-mentioned authors promote the ALL as a main anterolateral knee stabilizer, others refuted the relevance of the ALL in providing anterolateral knee stability [18] and put more emphasis on the role of the deep portions of the iliotibial tract (ITT). The deep portion of the ITT was first described in the 1960s by Kaplan et al. and consists of two distinct parts: the deep attachments of the ITT to the distal femur and the capsulo-osseous layer of the ITT [17, 23, 28, 38].

This polarity between the deep structures of the ITT and the ALL is also evident in the field of 'anterior cruciate ligament reconstruction with concomitant anterolateral extraarticular reconstruction'. While some authors have advocated reconstruction of the ACL in combination with anatomical ALL reconstruction [16, 34], others prefer extra-anatomical lateral tenodesis by inserting the graft more proximal at the femur and therefore tend to mimic the deep structures of the ITT rather than those of the ALL $[1,25]$. For a more personalized and tailored anatomical reconstruction special care has to be taken to identify the real extent of extraarticular concomitant injuries in order to repair or reconstruct this specific structure, e.g. ALL or deep ITT. However, previous research investigated only MRI visibility of the ALL, providing visibilities between $51 \%$ and $98 \%[3,14,37]$. To date no such investigations were performed for the deep structures of the ITT.

The aim of the present study was to assess the visibility and reproducibility of both the ALL and the deep structures of the ITT using magnetic resonance imaging. It was hypothesized that Cohen's kappa values for intraand inter-observer reproducibility would be seen to be above 0.70 , indicating substantial agreement between the ratings [22].

\section{Materials and methods}

Ethics approval was obtained from the Ethics Committee of the Medical University of Innsbruck. The retrospective analysis was conducted in accordance with the ethical standards of the Declaration of Helsinki.

MRI data from patients aged 18 to 40 obtained between January 2017 and December 2018 were retrospectively reviewed. Only healthy knees without any clinically or radiologically diagnosed intra- or extraarticular ligamentous pathology were included. Further exclusion criteria were lesions to the joint capsule, fractures, bone edemas as well as foreign material and motion artifacts. Moreover, medical records were checked for past knee injuries or operative interventions.

All MRIs were obtained using the identical protocol. Patients were examined in supine position using a dedicated 15-channel knee coil. The following sequences were used for our $1.5 \mathrm{~T} / 3.0 \mathrm{~T}$ Scanner (Avanto/Skyra, Siemens, Erlangen, Germany): coronal T1-weighted images (TE 10/13, TR 696/522, SL $3 \mathrm{~mm}$ ); coronal PDweighted images with fat saturation (TE 40/38, TR 4100/3230, SL $3 \mathrm{~mm}$ ); sagittal PD-weighted images with fat saturation (TE 39/38, TR 3000/3710, SL 3 mm) and axial PD-weighted images with fat saturation (TE 31/37, TR 3010/3100, SL 3.5/3 mm).

Two trained and experienced musculoskeletal radiologists $(\mathrm{HB}, \mathrm{KC})$ analyzed the pictures using the imaging viewer Impax EE (Agfa Health Care N.V., Mortsel, Belgium). Before commencement, a specialist in the field of anterolateral knee anatomy lectured and briefed both radiologists in a private cadaver dissection class. All relevant lateral and anterolateral structures of the knee were dissected and studied.

Thereafter, each observer performed all the belowmentioned analyses separately. To allow calculation of intra-observer agreement, all measurements were performed twice at an interval greater than 2 weeks between evaluations.

The analysis to identify the ALL was performed in a manner similar to previous research $[14,37]$. The ALL was defined as a low-signal band with its origin at the postero-proximal region of the femoral epicondyle, running in an antero-distal direction deep to the ITT (with optional fibers to the lateral meniscus; bifurcation point), crossing the lateral collateral ligament in its proximal third and inserting on the anterolateral tibia midway between Gerdy's tubercle and the fibular head [8] (Fig. 1).

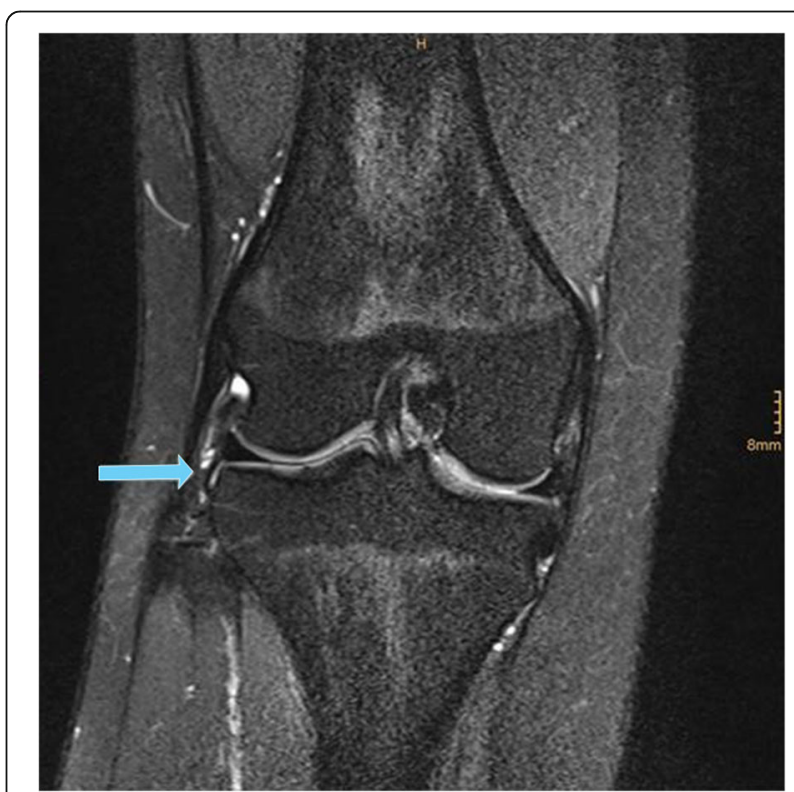

Fig. 1 Tibial part (blue arrow) of the anterolateral ligament (ALL) as seen in $73.2 \%$ of the examined cases (coronal PD-weighted sequence with fat saturation) 
Three parts of the ALL were defined and their presence assessed on a binary (yes/no) basis: a) femoral part, b) meniscal part, c) tibial part. The ALL was assessed only when present and clearly seen on both axial and coronal sequences under direct cross-referencing of images.

The deep attachments of the ITT at the distal femur were also evaluated on a binary (yes/no) basis and further subcategorized according to the literature in three sub-structures: insertion near the septum, supracondylar insertion and retrograde insertion $[8,17,23]$. Furthermore, the presence of the capsulo-osseus layer of the ITT, defined as deep fibers running from the region of the Kaplan fiber complex to the anterolateral tibia, was examined [8, 17, 23, 28, 38] (Fig. 2).

After data analysis, a consensus meeting of the two radiologists was conducted to discuss discrepant findings.

\section{Statistics}

Descriptive statistics for visibility of the above-mentioned structures are given for the consensus rating. Cohen's kappa and 95\% confidence intervals (95\% CI) were determined as a measure of inter- and intra-observer reproducibility. In addition, we calculated the agreement separately for the categories "visible" and "not visible" as suggested by Cicchetti and Feinstein [2]. A value of 0.70 for Cohen's kappa was deemed the threshold for substantial reproducibility [22]. Sample size considerations are based on a power analysis for a Pearson correlation as an approximation of Cohen's kappa coefficient. Power analysis for Cohen's kappa was not available in common power analysis software packages. An observed correlation

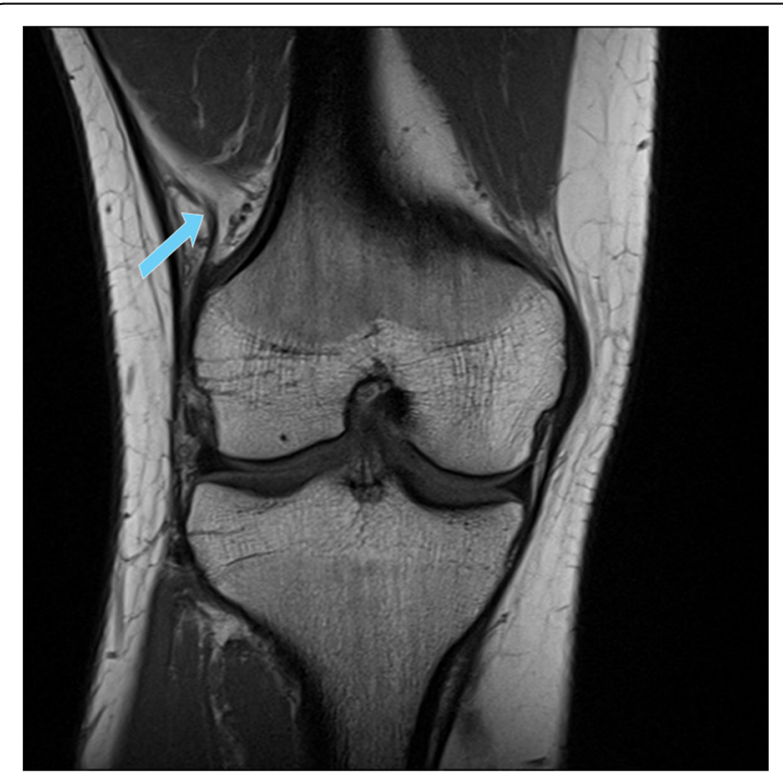

Fig. 2 Deep attachments of the iliotibial tract (ITT) to the distal femur (blue arrow) as seen in $60.6 \%$ of the examined cases (coronal $\mathrm{T} 1$-weighted sequence) coefficient of 0.824 in a sample of 70 cases was sufficient to demonstrate that the 0.70 threshold was exceeded with alpha $=0.05$ and beta $=0.20$ (one-sided). Power analysis was performed with $G$ *Power 3.1.9.2.

\section{Results}

Seventy-one patients (29 women, $40.8 \%$ ) with an average age of $44.7 \pm 14.1$ years met the inclusion criteria.

In $62.0 \%$ of the cases the femoral part of the ALL was visible, whereas visibility of the meniscal and the tibial part of the ALL was $0 \%$ and $73.2 \%$, respectively. The deep attachments of the ITT to the distal femur were detected in $60.6 \%$ of the cases. However, in none of the patients was it possible to further distinguish these fibers as 'insertion near septum', 'supracondylar insertion' or 'retrograde insertion'. Visibility of the capsulo-osseous layer of the ITT was $0 \%$.

Inter-observer agreement was high. Cohen's kappa was 0.97 (95\%CI: 0.9-1.00) for the tibial part of the ALL and 0.76 (95\%CI: 0.61-0.92) for the femoral part of the ALL. As previously mentioned, neither of the two observers was able to detect the meniscal part of the ALL in any of the patients (100\% absolute agreement). Therefore, it was not possible to calculate Cohen's kappa for this variable. For the deep attachments of the ITT to the distal femur Cohen's kappa was 0.94 (95\%CI: 0.86-1.00). As the capsulo-osseous layer of the ITT was not visible to either of the observers in $100 \%$ of the cases, it was also not possible to calculate Cohen's kappa for that parameter. For each of the investigated parameters absolute agreement between the observers was at least $88 \%$. For further details, see Table 1.

Regarding intra-observer agreement, Cohen's kappa was 0.62 (95\%CI: 0.43-0.81) for the femoral part of the ALL, 0.85 (95\%CI: 0.70-0.91) for the tibial part of the ALL and not quantifiable for the meniscal ALL part. For the deep attachments of the ITT to the distal femur Cohen's kappa was 0.94 (95\%CI: 0.86-1.00). As the capsulo-osseous layer of the ITT was not visible in 100\% of the cases, it was also not possible to calculate Cohen's kappa for that parameter. For each of the investigated parameters absolute agreement between the two time points was at least $83 \%$ (Table 1).

\section{Discussion}

The primary finding of the present study is good MRI visibility of the ALL and the deep fibers of the ITT with substantial and inter- and intra-observer agreement. The femoral and tibial parts of the ALL were visible in $62 \%$ and $73.2 \%$ of the cases, respectively, whereas the deep attachment of the ITT to the distal femur was detected in $60.6 \%$ of the cases. The capsulo-osseous layer of the ITT could not be assessed in any of the cases. 
Table 1 Inter- and intra-observer reproducibility results for the different parts of the ALL and the deep ITT structures

\begin{tabular}{|c|c|c|c|c|c|}
\hline & \multirow{2}{*}{$\begin{array}{l}\text { Visibility } \\
\%\end{array}$} & \multicolumn{2}{|l|}{ Inter-observer } & \multicolumn{2}{|l|}{ Intra-observer } \\
\hline & & Cohen's kappa $(95 \%$ Cl) & $\begin{array}{l}\text { Absolute Agreement [\%]": } \\
\text { Total / not visible/visible }\end{array}$ & Cohen's kappa $(95 \%$ Cl) & $\begin{array}{l}\text { Absolute Agreement [\%] } \\
\text { Total / not visible / visible }\end{array}$ \\
\hline Femoral part ALL & 62.0 & $0.76(0.61-0.92)$ & $88.7 / 85.2 / 90.9$ & $0.62(0.43-0.81)$ & $83.1 / 75.0 / 87.2$ \\
\hline Meniscal part ALL & 0.0 & n.c. & $100 / 100 / 100$ & n.c. & $100 / 100 / 100$ \\
\hline Tibial part ALL & 73.2 & $0.97(0.9-1.00)$ & $98.6 / 97.4$ / 99.0 & $\begin{array}{l}0.85(0.70-0.91) \\
0.846(0.701-0.911)\end{array}$ & $94.4 / 88.2$ / 96.3 \\
\hline $\begin{array}{l}\text { Deep attachments of the ITT to } \\
\text { the distal femur }\end{array}$ & 60.6 & $0.94(0.86-1.00)$ & 97.2 / 96.4 / 97.7 & $0.94(0.86-1.00)$ & 97.2 / 96.3 / 97.7 \\
\hline Capsulo-osseous layer of the ITT & 0.0 & n.c. & $100 / 100 / 100$ & n.c. & $100 / 100 / 100$ \\
\hline
\end{tabular}

Several previous studies have dealt with MRI visibility of the ALL $[9,12-14,20,21,24,30,37]$. However only limited knowledge exists about inter-observer reproducibility [7, 13, 14, 37]. Inter- and intra-observer reliabilities of the ALL have been reported to range between $0.69-1.00$ and $06-1.00$, respectively [7, 13]. These results are in line with the present inter- and intra-observer agreements of $88 \%$ and $83 \%$. In addition to the visibility of the different structures, an attempt was made to compare the descriptive values to the different parts of the ALL and the findings made in the above-mentioned studies. However, only three studies differentiated between the different parts of the ALL [12, 14, 24]. Visibility of the femoral (62\%) and tibial part (73.2\%) of the ALL was well consistent with previous studies. However, compared to the above-mentioned studies, the meniscal part of the ALL was detected less frequently. The reason for this discrepancy remains unclear. Other previous studies dealt with the distinct subject of ALL co-injury rates in patients with ACL rupture. However, this is beyond the scope of the present investigation $[3,10,15,27]$.

To the best of our knowledge, there exist no previous studies that deal with MRI visibility of the deep structures of the ITT. This is somewhat surprising because these structures were described from an anatomic and a biomechanical point of view many years ago and were considered important for anterolateral knee stability $[17,23,28,38]$. In the present study, substantial agreement was found for MRI visibility of the deep structures of the ITT. The deep attachment of the ITT to the distal femur was detected in $60.6 \%$ of the cases. It was not possible to further distinguish these fibers as 'insertion near septum', 'supracondylar insertion' or 'retrograde insertion', as defined by Lobenhoffer [23]. It is noteworthy that in the present investigation the capsulo-osseous layer of the ITT could not be assessed in any of the cases. A possible explanation for this undifferentiability might be the immediate close proximity of many other structures like the superficial ITT or the tibial part of the ALL at the proximal anterolateral tibia. It seems reasonable that the limitations in the spatial resolution of MRI as compared with anatomic dissection [30] inhibit sufficient discrimination between a) the distal ALL, b) the superficial ITT and c) the capsulo-osseous layer of the ITT.

This work has some limitations. This was a retrospective investigation with the weaknesses typically associated with such studies. Although strict inclusion and exclusion criteria were applied, it would have been even better to include subjects $100 \%$ free of knee complaints. The subjects included in the present retrospective analysis were free of major knee lesions, but must have had some knee complaints as a prerequisite for MRI examination.

The major strength of the present study is that MRI visibility was investigated for the first time for both the ALL and the deep structures of the ITT in one single study, including a large cohort of patients and using a 3 Tesla MRI. Moreover, inter- and intra-observer reproducibility, which have not been reported in previous studies, were assessed. Compared to other studies, only healthy participants with no ligamentous lesions where included and examined.

Since the assessment and subsequently the treatment of an injured structure is preferably done after correct radiological representation, the clinical value of the present data is high. This is especially true, since injuries of the anterolateral structures are often seen with concomitant ACL ruptures [6]. Whether and how the anterolateral structures should be addressed at the time of ACL reconstruction is the subject of ongoing discussion $[1,8,16,25,34]$. Taken together the results of the present study substantially expand the current scientific insights into MRI visibility of the anterolateral structures of the knee and increase knowledge and safety in the field of anterolateral knee reconstruction. 


\section{Conclusion}

It can be concluded that the presence of the anterolateral structures of the knee can be determined using MRI with substantial inter- and intra-observer agreement. This is true for both the ALL and the deep structures of the ITT, but not for the capsule-osseous layer.

\section{Abbreviations}

ITT: Iliotibial Tract; ALL: Anterolateral Ligament; MRI: Magnetic Resonance Imaging

\section{Acknowledgments}

Professional native-speaker language editing by M.H. Margreiter is acknowledged.

\section{Authors' contributions}

ML: Concept, writing. AR: Writing, critical review. BH: Measurements. CK: Measurements. CK: Writing, critical review. WH: Critical review. JG: Statistics. The author(s) read and approved the final manuscript.

\section{Funding}

No funding.

\section{Availability of data and materials}

The datasets used and/or analysed during the current study are available from the corresponding author on reasonable request.

\section{Ethics approval and consent to participate}

Ethics approval was obtained from the Ethics Committee of the Medical University of Innsbruck (Ethikkommission der Medizinischen Universität Innsbruck)

Professional language editing performed by Mary Heaney Margreiter, professional translator/editor and Certified Court Interpreter.

\section{Consent for publication}

Not applicable.

\section{Competing interests}

All authors declare that they have no competing interests.

\section{Author details}

'Deptartment of Orthopedic Surgery, Medical University of Innsbruck, Innsbruck, Austria. ${ }^{2}$ Deptartment of Trauma Surgery, Medical University of Innsbruck, Innsbruck, Austria. ${ }^{3}$ Department of Radiology, Medical University of Innsbruck, Innsbruck, Austria. ${ }^{4}$ Medical University of Innsbruck, Innsbruck, Austria. ${ }^{5}$ Innsbruck Institute of Patient-centered Outcome Research (IIPCOR) Innsbruck, Austria.

\section{Received: 2 January 2020 Accepted: 21 April 2020}

\section{Published online: 06 May 2020}

\section{References}

1. Buda R, Ruffilli A, Di Caprio F et al (2013) Allograft salvage procedure in multiple-revision anterior cruciate ligament reconstruction. Am J Sports Med 41:402-410

2. Cicchetti DV, Feinstein AR (1990) High agreement but low kappa: II. Resolving the paradoxes. J Clin Epidemiol 43:551-558

3. Claes S, Bartholomeeusen S, Bellemans J (2014) High prevalence of anterolateral ligament abnormalities in magnetic resonance images of anterior cruciate ligament-injured knees. Acta Orthop Belg 80:45-49

4. Claes S, Vereecke E, Maes M et al (2013) Anatomy of the anterolateral ligament of the knee. J Anat 223:321-328

5. Dodds AL, Halewood C, Gupte CM et al (2014) The anterolateral ligament: anatomy, length changes and association with the Segond fracture. Bone Joint J 96-B:325-331

6. Ferretti A, Monaco E, Fabbri M et al (2017) Prevalence and classification of injuries of anterolateral complex in acute anterior cruciate ligament tears. Arthroscopy 33:147-154

7. Ferretti A, Monaco E, Redler A et al (2019) High prevalence of anterolateral ligament abnormalities on MRI in knees with acute anterior cruciate ligament injuries: a case-control series from the SANTI study group. Orthop J Sports Med 7:2325967119852916

8. Getgood A, Brown C, Lording T et al (2019) The anterolateral complex of the knee: results from the international ALC consensus group meeting. Knee Surg Sports Traumatol Arthrosc 27:166-176

9. Gossner J (2014) The anterolateral ligament of the knee - visibility on magnetic resonance imaging. Rev Bras Ortop 49:98-99

10. Hartigan DE, Carroll KW, Kosarek FJ et al (2016) Visibility of anterolateral ligament tears in anterior cruciate ligament-deficient knees with standard 1. 5-tesla magnetic resonance imaging. Arthroscopy 32:2061-2065

11. Helito CP, Demange MK, Bonadio MB et al (2013) Anatomy and histology of the knee anterolateral ligament. Orthop J Sports Med 1:2325967113513546

12. Helito CP, Demange MK, Helito PV et al (2015) Evaluation of the anterolateral ligament of the knee by means of magnetic resonance examination. Rev Bras Ortop 50:214-219

13. Helito CP, Helito PV, Bonadio MB et al (2015) Correlation of magnetic resonance imaging with knee anterolateral ligament anatomy: a cadaveric study. Orthop J Sports Med 3:2325967115621024

14. Helito CP, Helito PV Costa HP et al (2014) MRI evaluation of the anterolateral ligament of the knee: assessment in routine 1.5-T scans. Skelet Radiol 43:1421-1427

15. Helito CP, Helito PV, Costa HP et al (2017) Assessment of the anterolateral ligament of the knee by magnetic resonance imaging in acute injuries of the anterior cruciate ligament. Arthroscopy 33:140-146

16. Ibrahim SA, Shohdy EM, Marwan Y et al (2017) Anatomic reconstruction of the anterior cruciate ligament of the knee with or without reconstruction of the anterolateral ligament. T Am J Sports Med 45:363546517691517

17. Kaplan EB (1958) The iliotibial tract; clinical and morphological significance. J Bone Joint Surg Am 40-A:817-832

18. Kittl C, El-Daou H, Athwal KK et al (2016) The role of the anterolateral structures and the ACL in controlling laxity of the intact and $A C L$-deficient knee. Am J Sports Med 44:345-354

19. Kittl C, El-Daou H, Athwal KK et al (2016) The role of the anterolateral structures and the ACL in controlling laxity of the intact and $A C L$-deficient knee: response. Am J Sports Med 44:NP15-NP18

20. Klontzas ME, Maris TG, Zibis AH et al (2016) Normal magnetic resonance imaging anatomy of the anterolateral knee ligament with a T2/T1-weighted 3-dimensional sequence: a feasibility study. Can Assoc Radiol J 67:52-59

21. Kosy JD, Mandalia VI, Anaspure R (2015) Characterization of the anatomy of the anterolateral ligament of the knee using magnetic resonance imaging. Skelet Radiol 44:1647-1653

22. Landis JR, Koch GG (1977) The measurement of observer agreement for categorical data. Biometrics 33:159-174

23. Lobenhoffer P, Posel P, Witt $S$ et al (1987) Distal femoral fixation of the iliotibial tract. Arch Orthop Trauma Surg 106:285-290

24. Macchi V, Porzionato A, Morra A et al (2016) The anterolateral ligament of the knee: a radiologic and histotopographic study. Surg Radiol Anat 38:341348

25. Marcacci M, Zaffagnini S, Giordano G et al (2009) Anterior cruciate ligament reconstruction associated with extra-articular tenodesis: a prospective clinical and radiographic evaluation with 10- to 13-year follow-up. Am J Sports Med 37:707-714

26. Monaco E, Ferretti A, Labianca L et al (2012) Navigated knee kinematics after cutting of the $A C L$ and its secondary restraint. Knee Surg Sports Traumatol Arthrosc 20:870-877

27. Monaco E, Helito CP, Redler A et al (2019) Correlation between magnetic resonance imaging and surgical exploration of the anterolateral structures of the acute anterior cruciate ligament-injured knee. Am J Sports Med 47: 1186-1193

28. Müller W (1983) The knee: form, function and ligament reconstruction. Springer, Berlin

29. Parsons EM, Gee AO, Spiekerman C et al (2015) The biomechanical function of the anterolateral ligament of the knee. Am J Sports Med 43:669-674

30. Porrino J Jr, Maloney E, Richardson M et al (2015) The anterolateral ligament of the knee: MRI appearance, association with the Segond fracture, and historical perspective. AJR Am J Roentgenol 204:367-373

31. Rahnemai-Azar AA, Miller RM, Guenther D et al (2016) Structural properties of the anterolateral capsule and Iliotibial band of the knee. Am J Sports Med 44:892-897

32. Runer A, Birkmaier S, Pamminger $M$ et al (2016) The anterolateral ligament of the knee: a dissection study. Knee 23:8-12 
33. Saiegh YA, Suero EM, Guenther D et al (2015) Sectioning the anterolateral ligament did not increase tibiofemoral translation or rotation in an ACLdeficient cadaveric model. Knee Surg Sports Traumatol Arthrosc 25:10861092

34. Sonnery-Cottet B, Thaunat M, Freychet B et al (2015) Outcome of a combined anterior cruciate ligament and anterolateral ligament reconstruction technique with a minimum 2-year follow-up. Am J Sports Med 43:1598-1605

35. Spencer L, Burkhart TA, Tran MN et al (2015) Biomechanical analysis of simulated clinical testing and reconstruction of the anterolateral ligament of the knee. Am J Sports Med 43:2189-2197

36. Stijak L, Bumbasirevic M, Radonjic V et al (2016) Anatomic description of the anterolateral ligament of the knee. Knee Surg Sports Traumatol Arthrosc 24: 2083-2088

37. Taneja AK, Miranda FC, Braga CA et al (2015) MRI features of the anterolateral ligament of the knee. Skelet Radiol 44:403-410

38. Terry GC, Norwood LA, Hughston JC et al (1993) How iliotibial tract injuries of the knee combine with acute anterior cruciate ligament tears to influence abnormal anterior tibial displacement. Am J Sports Med 21:55-60

\section{Publisher's Note}

Springer Nature remains neutral with regard to jurisdictional claims in published maps and institutional affiliations.

\section{Submit your manuscript to a SpringerOpen ${ }^{\odot}$ journal and benefit from:}

- Convenient online submission

- Rigorous peer review

- Open access: articles freely available online

- High visibility within the field

- Retaining the copyright to your article

Submit your next manuscript at $\boldsymbol{\nabla}$ springeropen.com 